\title{
Recurrence of pre-eclampsia and the risk of future hypertension and cardiovascular disease: a systematic review and meta-analysis
}

\author{
L Brouwers, ${ }^{a}$ AJ van der Meiden-van Roest, ${ }^{a}$ C Savelkoul, ${ }^{a}$ TE Vogelvang, ${ }^{b}$ AT Lely, ${ }^{a}$ A Franx, ${ }^{a}$ \\ BB van Rijn ${ }^{a}$
}

${ }^{a}$ Department of Obstetrics, Wilhelmina Children's Hospital Birth Centre, University Medical Centre Utrecht, Utrecht University, Utrecht, the Netherlands ${ }^{\mathrm{b}}$ Department of Obstetrics and Gynaecology, Diakonessenhuis, Utrecht, the Netherlands

Correspondence: L Brouwers, Department of Obstetrics, Wilhelmina Children's Hospital Birth Centre, University Medical Centre Utrecht, Utrecht University, Lundlaan 6, PO Box 85090, 3508 AB Utrecht, the Netherlands. Email: 1.brouwers-6@umcutrecht.nl

Accepted 22 June 2018. Published Online 9 August 2018.

This paper includes Author Insights, a video abstract available at https://vimeo.com/rcog/authorinsights15394

Background Women with a history of hypertensive disorders, including pre-eclampsia, during pregnancy have a two- to-fivefold increased risk of cardiovascular disease (CVD). In 15\% of women, pre-eclampsia recurs in the following pregnancy.

Objectives To evaluate all evidence on the future risk of developing hypertension and CVD after multiple pregnancies complicated by pre-eclampsia compared with pre-eclampsia in a single pregnancy followed by normal subsequent pregnancy.

Search strategy Embase and Medline were searched until June 2017.

Selection criteria All relevant studies on the risk of developing hypertension, atherosclerosis, ischaemic heart disease, cerebrovascular accident (CVA), thromboembolism, heart failure or overall hospitalisation and mortality due to CVD after having had recurrent pre-eclampsia.

Data collection and analysis Twenty-two studies were included in the review. When possible, we calculated pooled risk ratios (RR) with $95 \%$ CI through random-effect analysis.

Main results Recurrent pre-eclampsia was consistently associated with an increased pooled risk ratio of hypertension (RR 2.3; 95\%
CI 1.9-2.9), ischaemic heart disease (RR 2.4; 95\% CI 2.2-2.7), heart failure (RR 2.9; 95\% CI 2.3-3.7), CVA (RR 1.7; 95\% CI 1.22.6) and hospitalisation due to CVD (RR 1.6; 95\% CI 1.3-1.9) when compared with women with subsequent uncomplicated pregnancies. Other studies on thromboembolism, atherosclerosis and cardiovascular mortality found a positive effect, but data could not be pooled.

Conclusions This systematic review and meta-analysis support consistent higher risk for future development of hypertension and CVD in women with recurring pre-eclampsia as opposed to women with a single episode of pre-eclampsia.

Keywords Cardiovascular disease, hypertension, long-term maternal outcomes, pre-eclampsia, recurrence.

Tweetable abstract The risk of future cardiovascular disease increases when women have recurrence of pre-eclampsia compared with a single episode.

Linked article This article is commented on by LH Theilen, p. 1655 in this issue. To view this mini commentary visit https:// doi.org/10.1111/1471-0528.15425.

Please cite this paper as: Brouwers L, van der Meiden-van Roest AJ, Savelkoul C, Vogelvang TE, Lely AT, Franx A, van Rijn BB. Recurrence of pre-eclampsia and the risk of future hypertension and cardiovascular disease: a systematic review and meta-analysis. BJOG 2018;125:1642-1654.

\section{Introduction}

At present, the World Health Organization states that one in five women suffer from hypertension and almost half of mortality in women is caused by cardiovascular disease (CVD). ${ }^{1,2}$ In the past decades, large cohort studies have consistently shown an increased association of CVD in women with a history of pre-eclampsia compared with women with uncomplicated pregnancies. ${ }^{3,4}$ This has led to a better understanding of female-specific risk factors for developing CVD. ${ }^{5,6}$ Pre-eclampsia complicates 3-5\% of first pregnancies and recurs in approximately $15 \%$ of subsequent pregnancies. ${ }^{3,7,8}$ Common underlying risk factors such as obesity, dyslipidaemia, inflammatory pathways and endothelial dysfunction are thought to contribute to both CVD and pre-eclampsia-complicated pregnancies. ${ }^{9-12}$ As 
pregnancy requires comprehensive physiological changes in the endocrine, respiratory and circulatory systems, a complicated pregnancy may reveal a predisposition to CVD and act as a 'stress test' identifying women at risk for future disease. Whether metabolic and cardiovascular changes induced by pre-eclampsia independently create a higher risk of CVD remains unknown.

Some countries have recently started notifying formerly pre-eclamptic women of their increased risks of CVD and advise women to actively test for modifiable risk factors at an early age. ${ }^{13-16}$ Pre-emptive screening, early recognition and treatment may prove to be useful in preventing longterm morbidity and mortality. ${ }^{1}$

It is well known that women with pre-eclampsia in their first pregnancy tend to have a milder variant or no disease in following pregnancies. ${ }^{8}$ It is conceivable that women who experience multiple episodes of pre-eclampsia fail to adjust to the physiological changes and physical stresses more than women with subsequent uncomplicated pregnancies. As not all women with pre-eclampsia develop CVD later in life, the recurrence of disease may be a helpful indicator for the necessity of screening. Previous studies briefly mention an effect of multiple pre-eclampsia-affected pregnancies on the risk of future hypertension and CVD. Although several reviews have been conducted on the relationship between pregnancy outcome and CVD, to the best of our knowledge, no systematic review or meta-analysis has been conducted on recurrent pregnancy complications and future lifetime cardiovascular risk. This study aims to evaluate all available evidence on the effect that recurrent pre-eclampsia has on long-term CVD risk compared with a single episode of pre-eclampsia with subsequent uncomplicated pregnancy.

\section{Methods}

\section{Literature search}

Medline and Embase were searched (until 1 June 2017) using search terms for 'pre-eclampsia', 'hypertension' and 'cardiovascular disease'. We restricted the search to various synonyms for 'recurrent', 'follow up', 'risk' and 'history', as many articles have been published on CVD after preeclampsia. A detailed description of the search strategy can be found in the Supplementary material (Appendix S1). Reference lists of original and review articles were reviewed. Articles in languages other than English or Dutch were translated using Google Translate and included when translation quality was sufficient. Unpublished studies were not included. The core outcome set for CVD after pregnancy complications (COMET registration number 701) is currently being developed and could not be used for this systematic review. There was no patient or public involvement in the carrying out of this study.

\section{Inclusion and exclusion criteria}

Inclusion criteria were as follows: (1) original articles; (2) studies that compared women with recurrent pre-eclampsia to women with a single episode of pre-eclampsia followed by uneventful pregnancies; (3) cerebrovascular accident (CVA), ischaemic heart disease (IHD), thromboembolism, atherosclerosis, heart failure, CVD mortality, hypertension or cardiovascular hospitalisation as outcome; (4) full-length article available; (5) inclusion of more than ten women; and (6) adult population. Studies with a follow-up duration of $<1$ year were excluded. We only included studies in which specific data on pre-eclampsia could be subtracted.

\section{Study selection and data extraction}

Two reviewers (LB, and CS or AJM) independently reviewed the title/abstract of all potential studies. As most articles do not show data on recurrence in their respective abstracts, many were reviewed as full text. Disagreement was resolved by discussion and consensus; if needed, the opinion of a third reviewer was decisive. Authors of articles with a promising study set-up but no data in association with recurrence of pre-eclampsia were contacted through email and allotted 3 months for a response. Data were extracted from each paper independently and included all relevant study specifics (i.e. definition of pre-eclampsia, follow-up time, outcome measures).

\section{Assessment of study quality and bias}

The methodological quality of studies was assessed using the Newcastle-Ottawa quality assessment Scale for cohort and case-control by two researchers (LB/AJM) independently. The Newcastle-Ottawa Scale uses a scoring system of three categories: selection, comparability and outcome (cohort studies) or exposure (case-control studies). ${ }^{17}$ When a cohort study was based on one single cohort of women, the scale was adjusted accordingly. The Newcastle-Ottawa Scale scoring of each included study can be found in the Supplementary material (Table S1).

\section{Statistics}

Incidence numbers were extracted from the data reported in each paper. When extracting hazard ratios (HR) and odds ratios (ORs), the most complete multivariate models were used to adjust for potential confounders. Most studies compared single or multiple occurrence pre-eclampsiaaffected women to women without any complicated pregnancies, without making a comparison between the two affected groups. All hazard ratios described below are in comparison with women with solely uncomplicated pregnancy. We performed a meta-analysis to give a direct overview of the risk when comparing the groups among themselves. Reviewmanager 5.3.5 was used to calculate 
pooled risk ratios (RR) with 95\% CI using a randomeffects model. To measure the amount of between-study variation that is due to systematic heterogeneity rather than chance, the $I^{2}$ metric was used. We used the MOOSE checklist and PRISMA guidelines for this systematic review. ${ }^{18,19}$

This research did not receive any specific grant from funding agencies in the public, commercial or not-forprofit sectors.

\section{Results}

\section{Characteristics of the studies}

We identified 22 studies for this review, a detailed description of the selection process can be found in the Supplementary material (Figure S1). Tables 1 and 2 summarise the characteristics of the studies included for hypertension and cardiovascular morbidity and mortality, respectively. There was a large variance in sample sizes (28-1 108 581) and study populations were selected from all over the world with a majority from northwest Europe, Canada and the USA. Follow up ranged from 1 to 45 years. Most studies used the American Congress of Obstetricians and Gynecologists criteria to identify women with pre-eclampsia. The definition of hypertension as an outcome varied between measured mean blood pressure, antihypertensive treatment, hospital diagnosis, International Classification of Diseases (ICD) coding and self-reported disease or treatment. When looking at cardiovascular morbidity and mortality, the majority of studies used record linkage through ICD codes and death certificates.

\section{Quality of evidence}

The quality score of the included studies can be found in the Supplementary material (Table S1). Six studies reached the respective maximum of stars among the cohort and casecontrol studies (maximum nine stars) ${ }^{20-25}$; three studies received eight stars, ${ }^{26-28}$ two received seven, ${ }^{29,30}$ four received six, ${ }^{31-34}$ one received five $e^{35}$ and one study obtained three stars. $^{36}$ For studies comparing a single cohort, one study received the maximum score of seven stars. ${ }^{37}$ The remaining four studies received five of seven stars. ${ }^{38-41}$

\section{Hypertension}

Overall, 17 papers were found to report on developing hypertension after recurrent pre-eclampsia, details can be found in Table 1. Four studies reported on mean blood pressure after variable lengths of follow up..$^{22,28,36,37}$ Two studies performed their analysis 1-5 years postpartum and did not find any difference between the groups. ${ }^{28,37}$ Two studies followed women for almost two decades, finding a significant increase in mean blood pressure in the group with recurrent pre-eclampsia. ${ }^{22,36}$ Five studies found higher risk of antihypertensive medication use when pre-eclampsia was recurrent compared with a single complicated pregnancy and when compared with women with uncomplicated pregnancy only. ${ }^{20,22,24,38,39}$ Two smaller studies looked at the proportion of recurrent pre-eclampsia in women who were hypertensive at follow up after having had pre-eclampsia in the index pregnancy and found conflicting results. ${ }^{32,35}$ Three studies observed women who went on to have subsequent pregnancies after pre-eclampsia, in the first two articles a nonsignificant association was mentioned in the text. ${ }^{27,30,34}$ One study found a higher incidence of hypertension among women with recurring pre-eclampsia, although information on their study set-up was limited. ${ }^{34}$ Van Oostwaard et al. ${ }^{40,41}$ published on the risk of hypertension in women with recurrent pregnancyinduced hypertension or pre-eclampsia. The author kindly shared data regarding pre-eclampsia only, resulting in relatively small study groups. For women delivering at term $(n=74)$ and preterm $(n=59)$ there was a significantly higher chance of hypertension after recurrence of preeclampsia compared with women with a normal subsequent pregnancy (RR 1.83; 95\% CI 1.11-3.02 and RR 2.35; 95\% CI 1.02-5.43, respectively). Two groups performed large registry-based cohort studies with ICD codes comparing women with pre-eclampsia with women with only nonhypertensive pregnancy. $^{21,26}$ One study showed a risk gradient, with a higher risk of hypertension after preeclampsia in the first pregnancy (HR 2.70; 95\% CI 2.512.90) compared with women with two or more normotensive pregnancies. The risk increased ( $\mathrm{HR} 4.34 ; 95 \% \mathrm{CI}$ 3.98-4.74) for women with pre-eclampsia in their second pregnancy only, and increased again for women with preeclampsia in both pregnancies (HR 6.00; 95\% CI 5.40 $6.67) .{ }^{21}$ Auger et al. ${ }^{26}$ reported increasing hazard ratios of 3.7 (95\% CI 3.5-3.9) for a single episode of pre-eclampsia and 7.2 (95\% CI 6.6-7.8) for recurrent pre-eclampsia when compared with women who only had normotensive pregnancies, 25 years after the index pregnancy.

\section{Meta-analysis}

Incidence data on 52544 women could be extracted from seven studies to perform a meta-analysis. ${ }^{21,24,26,34,38,40,41}$ In the pooled analysis the risk ratio for hypertension after follow up was increased in women with recurrent pre-eclampsia (pooled RR 2.33; 95\% CI 1.86-2.92, Figure 1.1.1). Heterogeneity between studies was considerable $\left(I^{2}=82 \%\right)$, so a sensitivity analysis was performed. When excluding the largest study by Auger et al. from the pooled analysis, heterogeneity tested was lower $\left(I^{2}=1 \%\right)$, nonetheless the found effect did not change (pooled RR 2.57; 95\% CI 2.32-2.85). 


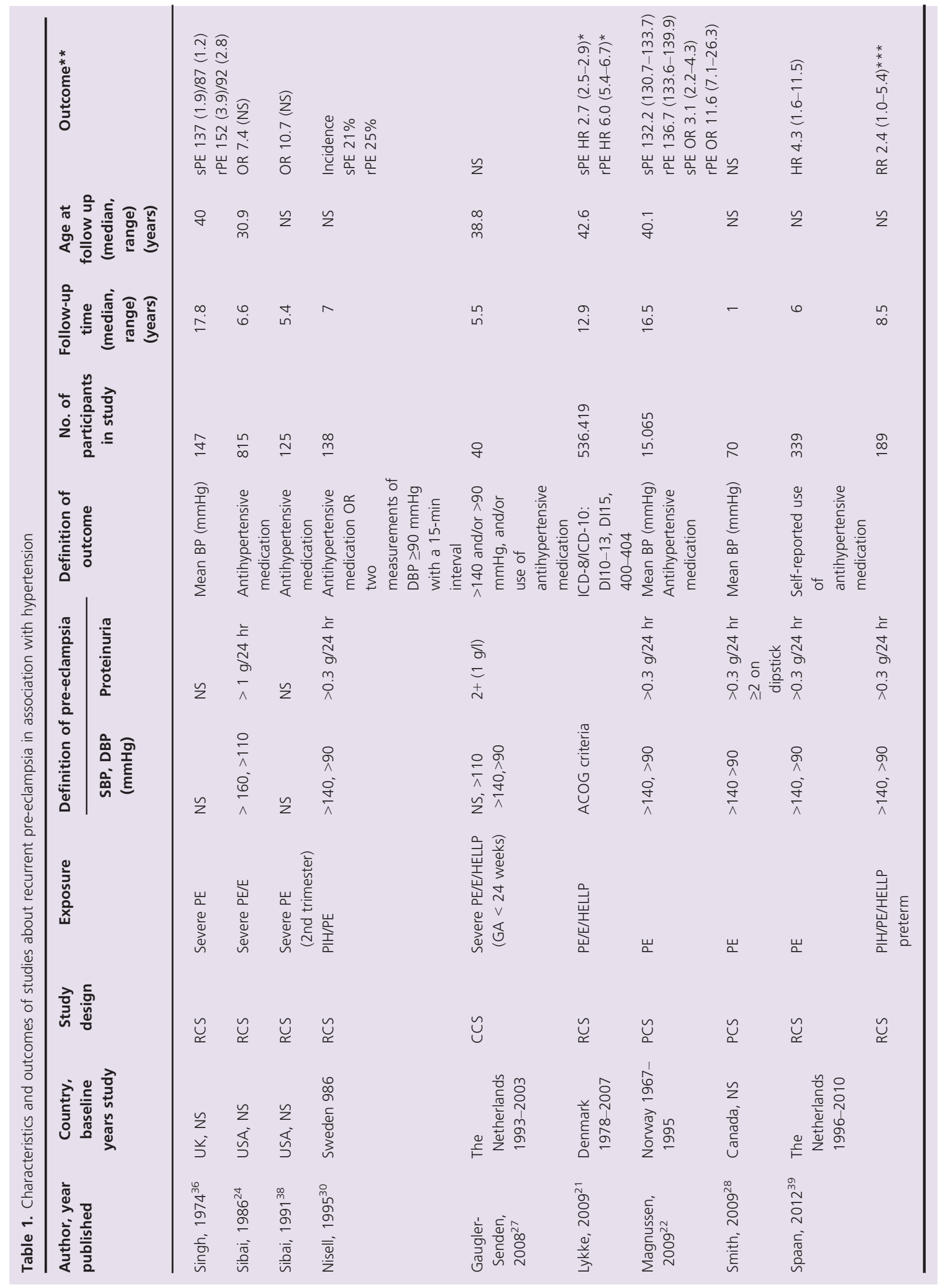




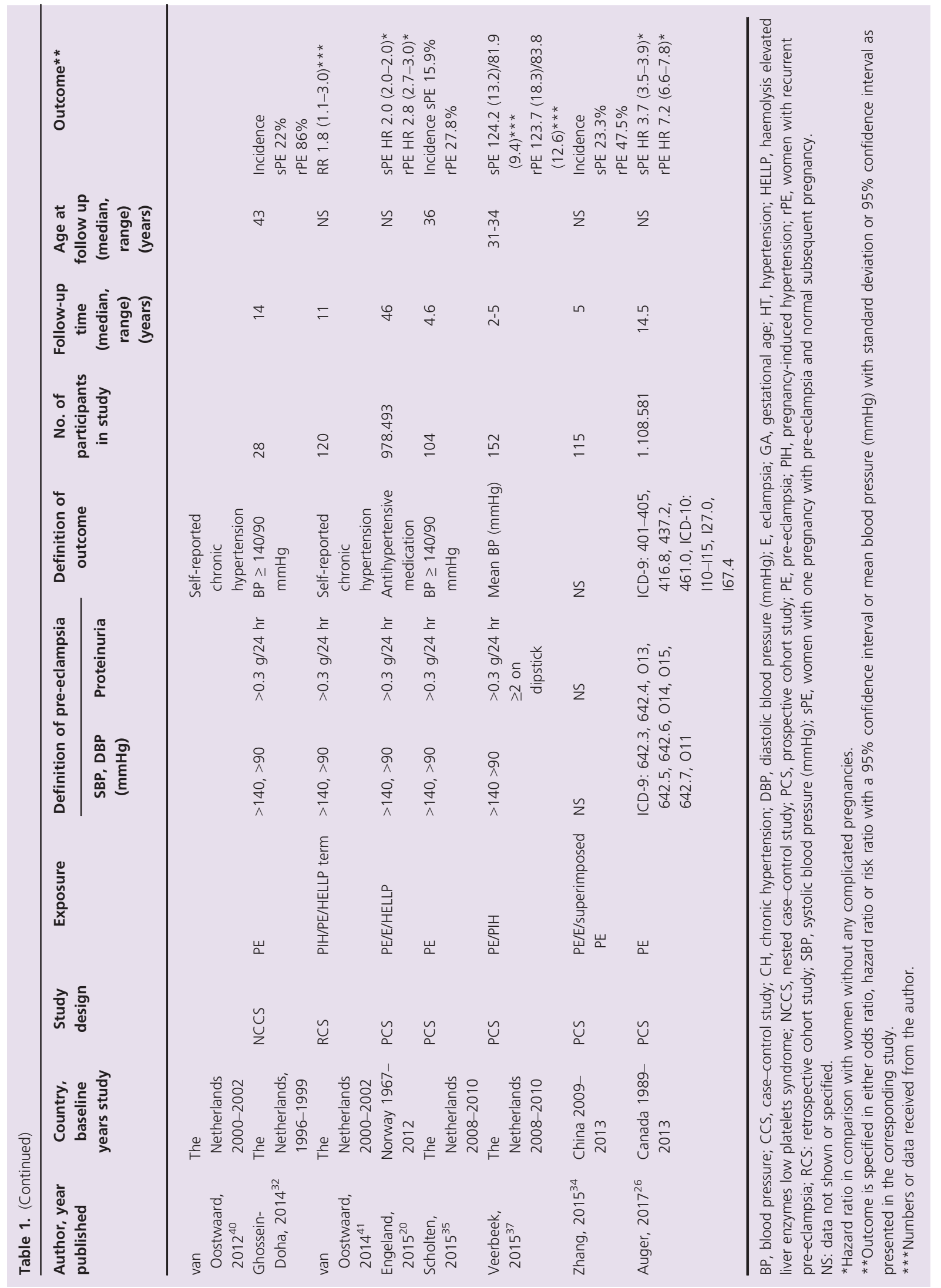




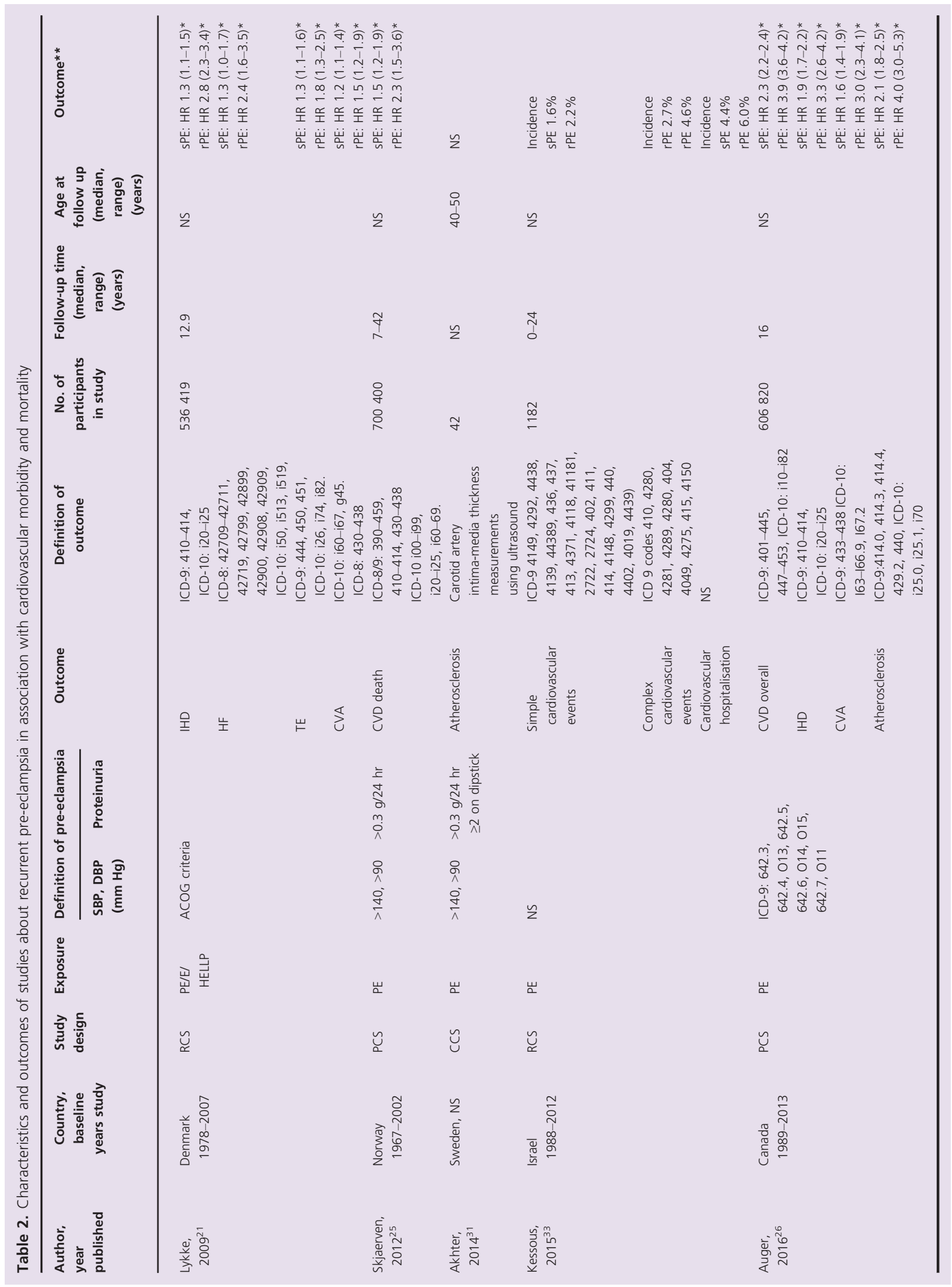




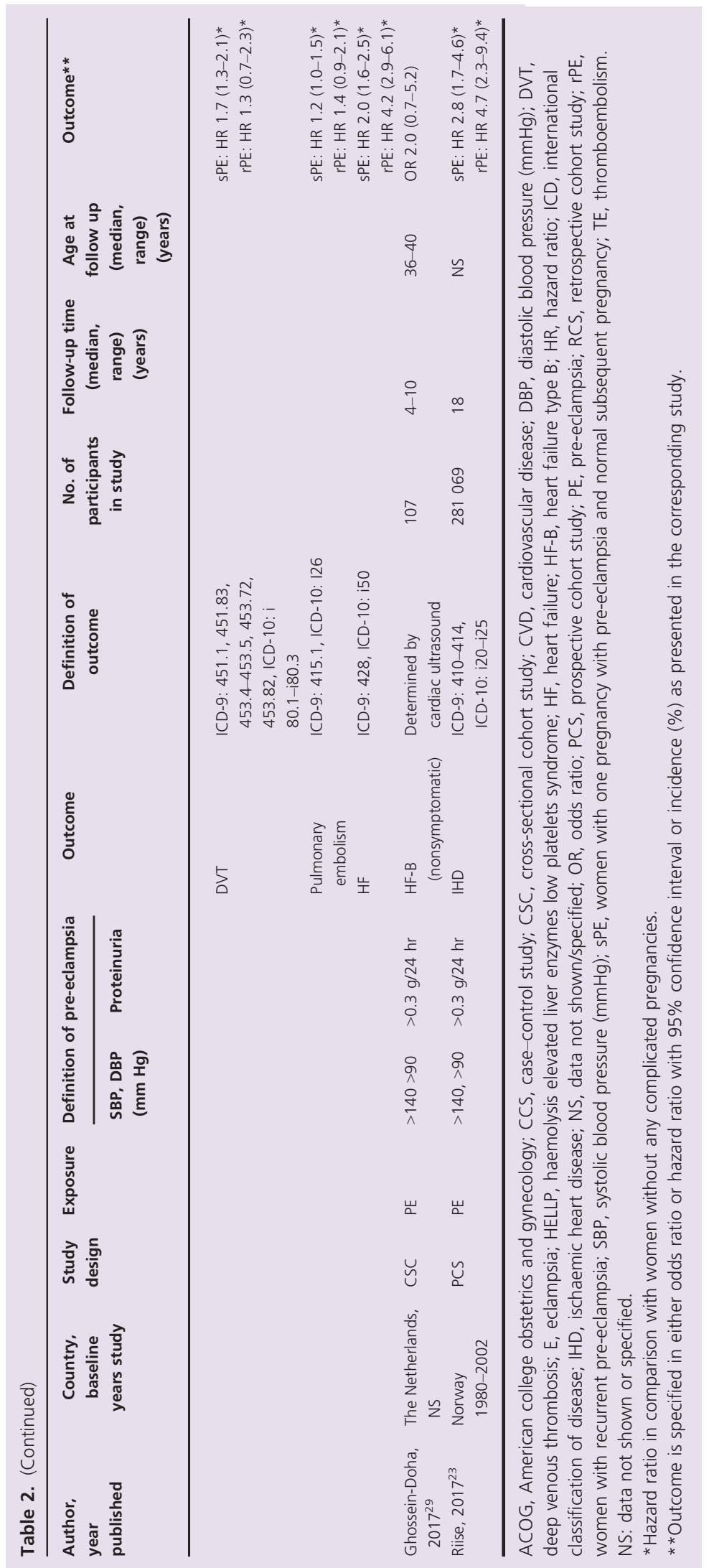




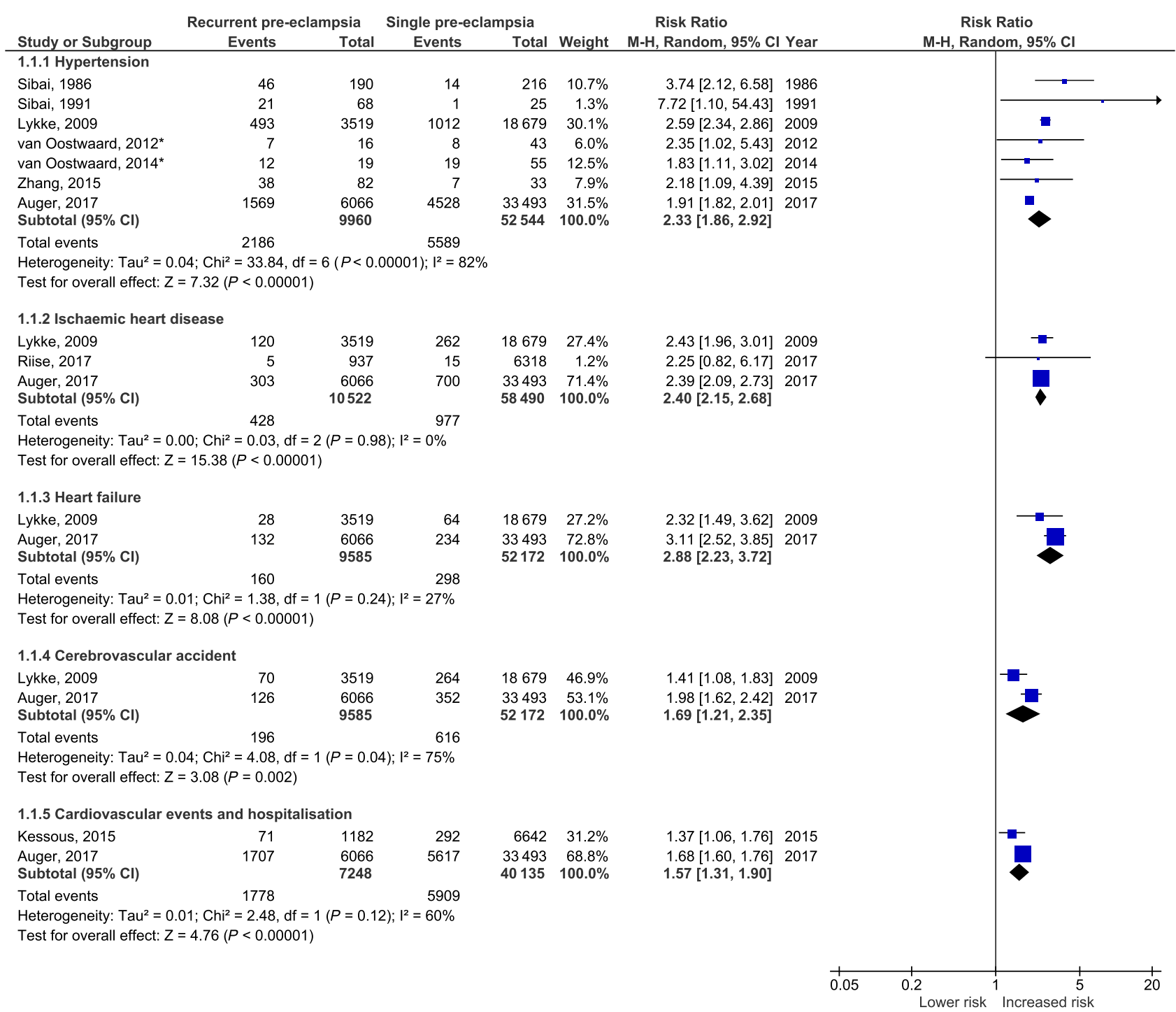

Figure 1. Forest plot of studies investigating the risk of hypertension (1.1.1), ischaemic heart disease (1.1.2), heart failure (1.1.3), cerebrovascular accident (1.1.4) and overall hospitalisation due to cardiovascular disease (1.1.5) after recurrent pre-eclampsia when compared with women with a single pregnancy affected by pre-eclampsia and subsequent normal pregnancy. Incidence data were extracted from original articles using available figures and tables. *Original data provided by author was used.

\section{Atherosclerosis}

Two studies reported on atherosclerosis after recurrent preeclampsia. ${ }^{26,31}$ An ICD-code for atherosclerosis was found to be significantly more present in the recurrent preeclampsia group (HR 4.0; 95\% CI 3.0-5.3) than in single affected women (HR 2.1; 95\% CI 1.8-2.5) when compared with women with solely uncomplicated pregnancies. ${ }^{26}$ Akhter et al. performed carotid artery intima-media thickness measurements in 42 women with previous pre-eclampsia. Although they found significantly higher intima-media thickness for women who had pre-eclampsia, they did not find a higher measurement when pre-eclampsia had recurred. ${ }^{31}$

\section{Thromboembolism}

Two record-linkage studies reported on various types of thrombosis after one or multiple pre-eclampsia-affected pregnancies compared with women with only uncomplicated pregnancy. ${ }^{21,26}$ One study discusses both deep venous thrombosis and pulmonary embolism in one category showing increasing hazard ratios when pre-eclampsia was recurrent. ${ }^{21}$ Auger et al. ${ }^{26}$ discussed results separately, 
finding higher hazard ratios for both outcomes when comparing single and recurrent pre-eclampsia with women with uncomplicated pregnancies (Table 2).

\section{Ischaemic heart disease}

Three record-linkage studies reported on IHD after one or multiple pre-eclampsia-affected pregnancies compared with women with only uncomplicated pregnancies. Riise et al. reported an increasing hazard ratio after recurrence of preeclampsia (HR 2.20; 95\% CI 0.91-5.32 in recurrent pre-eclampsia and HR 1.95; 95\% CI 1.31-2.91 for a single pre-eclampsia pregnancy), compared with unaffected pregnancies. When pre-eclampsia was combined with fetal growth restriction or preterm birth the change in hazard ratio was more significant (HR 4.66; 95\% CI 2.31-9.37 in recurrent pre-eclampsia as opposed to one episode of preeclampsia; HR 2.81; 95\% CI 1.70-4.61). ${ }^{23}$ The other two ICD-coded studies showed a similar increase in hazard ratio when comparing women with single or multiple affected pregnancies with women without pregnancy complications (Table 2). ${ }^{21,26}$

\section{Meta-analysis}

With all studies combined, 10522 women who had recurrent pre-eclampsia contributed to the meta-analysis on IHD. ${ }^{21,23,26}$ In the pooled analysis an increased risk of IHD was observed for recurrent pre-eclampsia (RR 2.40; 95\% CI 2.15-2.68; Figure 1.1.2). Heterogeneity between the studies was low $\left(I^{2}=0 \%\right)$.

\section{Heart failure}

Three studies described the development of heart failure. The two record-linkage studies mentioned above indicated higher hazard ratios for recurrent pre-eclampsia than for single pre-eclampsia-affected pregnancy compared with solely uncomplicated pregnancies (Table 2). ${ }^{21,26}$ GhosseinDoha et al. ${ }^{29}$ reported on (nonsymptomatic) heart failure type-B diagnosed by cardiac ultrasound 4-10 years postpartum. They did not find recurrence of pre-eclampsia to be significantly associated with this type of heart failure (OR 2.0; 95\% CI 0.7-5.2).

\section{Meta-analysis}

Due to the difference in outcome measures, only the data from two studies were comparable (Figure 1.1.3). ${ }^{21,26}$ In total, 9585 women had recurrent pre-eclampsia and showed a pooled risk ratio 2.88 (95\% CI 2.23-3.72). Heterogeneity was low $\left(I^{2}=27 \%\right)$.

\section{Cerebrovascular accident}

The same two record-linkage studies performed analysis on ischaemic and haemorrhagic CVA, finding higher adjusted hazard ratios for the women with recurrent pre-eclampsia than women with a single pregnancy with pre-eclampsia when compared with solely uncomplicated pregnancy (Table 2). ${ }^{21,26}$

Meta-analysis

When results of both studies were combined a risk ratio of 1.69 (95\% CI 1.21-2.35) was found with heterogeneity of $75 \%$ (Figure 1.1.4).

\section{Cardiovascular events and hospitalisation}

Kessous et al. performed a retrospective population study reporting on simple and complex cardiovascular events. Simple CVD events (i.e. hyperlipidaemia, hypertension) occurred significantly more in women with two or more pregnancies complicated by pre-eclampsia (2.2\% versus $1.6 \% ; P=0.001)$. Complex cardiovascular events (i.e. IHD, heart failure) occurred more frequently in the recurrent pre-eclampsia group compared with women with one pre-eclampsia-affected pregnancy $(4.6 \%$ versus $2.7 \% ; P=0.001)$. Patients were also admitted to the hospital more often due to CVD $(6.0 \%$ versus $4.0 \% ; P=0.001) .{ }^{33}$ This last outcome was also analysed in the record-linkage study by Auger et al., finding a similar trend [HR 3.9; 95\% CI 3.6-4.2 (recurrent pre-eclampsia) versus HR 2.3; 95\% CI 2.2-2.4 (single pre-eclampsia), compared with solely uncomplicated pregnancy]. ${ }^{26}$

\section{Meta-analysis}

When data were pooled for cardiovascular hospitalisations a pooled risk ratio of 1.57 (95\% CI 1.31-1.90) was found with some heterogeneity $\left(I^{2}=60 \%\right.$, Figure 1.1 .5$)$.

\section{Cardiovascular mortality}

Only one study analysed the association between recurrent pre-eclampsia and cardiovascular mortality. Even though they showed increasing hazard ratios for women with one, two or more pre-eclampsia-complicated pregnancies, there was no statistical significance when comparing the groups. $^{25}$

\section{Discussion}

\section{Main findings}

In this systematic review and meta-analysis we aimed to provide a comprehensive overview of available evidence on cardiovascular disease after recurrent pre-eclampsia. We found that women with recurrent pre-eclampsia have a threefold increased risk of heart failure, two- to three-fold risk of hypertension and IHD and almost a two-fold risk of CVA and overall CVD, when compared with women with a single event of pre-eclampsia and subsequent uncomplicated pregnancies. Although the set-up, size and quality of 
studies were variable, our pooled analysis indicated that the overall association between recurrent pre-eclampsia and CVD is a robust finding. As women with a history of preeclampsia have been shown to be at increased risk of CVD, this identifies a subgroup of women who are at even greater risk and could benefit from early preventive measures.

\section{Strengths and limitations}

This systematic review provides an overview of all available evidence up until June 2017. As most evidence on the risk of CVD after recurrent pre-eclampsia is based on small groups of women, the only way to obtain reliable results is by performing a meta-analysis. A random-effects model was used to incorporate between-study variation. We only included studies in which pre-eclampsia was clearly defined, leading to a clear and consistent additional risk of CVD in later life based on recurrence of this disease.

This study also has some limitations which need to be addressed. First, included studies date back to the 1970s and show a wide range of methodological quality. Only seven of 22 studies achieved the maximum score on the Newcastle-Ottawa Scale. Therefore, caution is needed when interpreting the results. Second, comparability between included studies is limited as definitions for exposure, outcome and effect measure differ. Consequently few data could be used for meta-analysis, possibly resulting in an over- or under-estimating of the risk when patient characteristics differ between and within studies. The small number of studies and the large population size of the main studies instantly lead to a higher heterogeneity in the metaanalysis. ${ }^{42}$ Also, different measurement of outcome, adjusting for confounders and duration of follow up, can lead to more variation than is to be expected. It could be argued that the addition of meta-analysis from a relatively small number of studies is not likely to improve accuracy in the effect estimates when findings are consistent within the studies, but does illustrate the continuing need for better original data. Third, we identified several studies in which analysis of recurrence of pre-eclampsia and development of CVD should be possible given the design of the study but was not mentioned in the paper. We experienced a low response rate to multiple emails to authors, hindering our inclusion of more studies. We speculate that it may be possible that some groups looked at this association, but found relatively small groups and minor correlations that were not important enough to mention, possibly leading to a form of publication bias. We believe it would be beneficial if more studies included recurrence of pre-eclampsia in their work to allow for improvement of our pooled estimated in the future. Finally, the larger registry-based studies used registered ICD codes upon discharge as outcome for hypertension and CVD. ${ }^{21-23,26}$ As the development of CVD and specifically hypertension does not always require hospitalisation, it is possible that only the most severe cases have been included, possibly leading to selection bias.

\section{Interpretation}

Several reviews and meta-analyses discuss the risk of CVD after pregnancy complications. ${ }^{3,4,6,43}$ Only a few discuss recurrence as a factor, usually stating a higher risk of CVD based on one or two studies. Mechanisms explaining the relation of CVD and pre-eclampsia are thought to be multifactorial. Several large studies have shown pre-eclampsia to be an independent risk factor when correcting for several established cardiovascular risk markers, such as hypertension. ${ }^{3}$ The significant correlation between pre-eclampsia, recurrence and the development of hypertension, results in our hypothesis that hypertension does not solely explain the association. We have yet to elucidate whether (recurrent) pre-eclamptic pregnancies induce metabolic and cardiovascular changes or if these women have a stronger predisposition for CVD. ${ }^{21,22,26,39}$

Other mechanisms that potentially plays a role in preeclampsia and CVD are of an inflammatory nature with chronic inflammatory risk markers being significantly higher in former pre-eclampsia patients. ${ }^{6,44}$ Pre-eclampsia and CVD also share other pathological features indicating similar pathways such as the presence of acute atherosis and endothelial cell dysfunction. ${ }^{45,46}$ All of the above strengthens the idea that pregnancy can be seen as a 'stress test' for cardiovascular health, identifying women at risk early in life.

Women with an early onset and/or severe pre-eclampsia are more likely to experience recurrence of disease compared with those who developed pre-eclampsia at term. ${ }^{7,8}$ Several studies found a consistently higher risk of cardiovascular morbidity and mortality when pre-eclampsia was early in onset or when combined with (iatrogenic) preterm birth or other complications like fetal growth restriction, irrespective of recurrence of pre-eclampsia. ${ }^{21,23,25}$ In one study from Riise et al., ${ }^{23}$ a steadily increasing risk of cardiovascular death after recurrent pre-eclampsia was found with higher hazard ratios when (recurrent) pre-eclampsia concurred with preterm delivery or fetal growth restriction. Unfortunately, there were no studies in which data on severity, time of onset and recurrence could be extracted for meta-analysis. ${ }^{3,33}$ Therefore, we cannot infer from our review to what extent the association between recurrent pre-eclampsia and CVD is explained by the onset and/or severity of the first episode.

Several studies within this review discussed timing as a factor in determining cardiovascular risk, morbidity and mortality. When looking at cardiovascular risk markers, studies found significant correlation after many years of follow up, even though these markers may not be apparent soon after pregnancy. ${ }^{27,28,30-32,35,37,40}$ A few studies analysed time between pre-eclampsia and CVD in their set-up, reporting a significantly shorter time to cardiovascular events 
in the recurrent group with a significantly accelerated disease progression. The magnitude of this time-specific association appears to decrease over time but remained significant when comparing recurrent and nonrecurrent pre-eclampsia. ${ }^{20,26}$ Several studies have shown that hypertension is present shortly after pre-eclampsia. ${ }^{47,48}$ After a longer latency period, age-specific risk factors might play a more prominent role and risk for hypertension and CVD in women with normotensive subsequent pregnancies will become more alike. However, studies with a longer follow up still showed significant increased risk in the recurrent group..$^{21,26,41}$ Some studies mention the high risk of having had pre-eclampsia among women having only one pregnancy. Possibly, women with the most severe form of pre-eclampsia refrain from subsequent pregnancies because of older age or perceived risk, preventing a dose-response type relationship from becoming apparent. ${ }^{23,25}$ There were several studies in our systematic search that did not specify the type of hypertensive pregnancy disorders and were therefore not included in this review. Interestingly, they report that women with recurrent gestational hypertension (including pre-eclampsia) have a similar increased risk of CVD. ${ }^{49-54}$

\section{Conclusion}

Evidence shows a strong relationship between recurrence of pre-eclampsia and additional risk of developing hypertension and CVD later in life compared with a single pregnancy with pre-eclampsia. With the increasing burden of CVD on society, this needs to be taken into consideration when establishing prevention programmes. This review shows that multiple complicated pregnancies may need to be weighed more heavily, compared with when subsequent pregnancies were normotensive.

\section{Disclosure of interests}

The authors declare that there is no conflict of interest. Completed disclosure of interests form available to view online as supporting information.

\section{Contribution to authorship}

$\mathrm{LB}, \mathrm{AJM}$ and CS were responsible for the acquisition and interpretation of the data. LB drafted the manuscript. AJM, $\mathrm{CS}$, TEV, ATL, AF and BBR edited and revised the manuscript. All authors have read and approved the final version of the manuscript.

\section{Details of ethics approval}

No approval of the institutional review board was required.

\section{Funding}

This research did not receive any specific grant from funding agencies in the public, commercial, or not-for-profit sectors.

\section{Acknowledgements}

The authors wish to thank all authors who collaborated and shared knowledge and data of previously published work for this systematic search and meta-analysis. In particular, we thank Jan Veerbeek and Miriam van Oostwaard for their collaboration on this paper.

\section{Supporting Information}

Additional supporting information may be found online in the Supporting Information section at the end of the article.

Figure S1. Flow chart of literature search for studies reporting on the association between recurrent pre-eclampsia and cardiovascular disease (CVD).

Table S1. Critical appraisal using the Newcastle-Ottawa Quality Assessment Scale for cohort and case-control studies. ${ }^{17}$

Appendix S1. Search strings.

Video S1. Author insights.

\section{References}

1 World Health Organization.. Women's health. Fact sheet N³34. 2013 September 2013. [http://www.who.int/mediacentre/factsheets/ fs334/en/] Accessed 11 July 2017

2 World Health Organization. Cardiovascular diseases (CVDs). Factsheet 2017 May 2017. [http://www.who.int/mediacentre/fac tsheets/fs317/en/] Accessed 11 July 2017.

3 Bellamy L, Casas JP, Hingorani AD, Williams DJ. Pre-eclampsia and risk of cardiovascular disease and cancer in later life: systematic review and meta-analysis. BMJ (Clinical research ed). 2007;335:974.

4 Brown MC, Best KE, Pearce MS, Waugh J, Robson SC, Bell R. Cardiovascular disease risk in women with pre-eclampsia: systematic review and meta-analysis. Eur J Epidemiol 2013;28:1-19.

5 Appelman Y, van Rijn BB, Ten Haaf ME, Boersma E, Peters SA. Sex differences in cardiovascular risk factors and disease prevention. Atherosclerosis 2015;241:211-8.

6 Zoet GA, Koster MP, Velthuis BK, de Groot CJ, Maas AH, Fauser $B C$, et al. Determinants of future cardiovascular health in women with a history of preeclampsia. Maturitas 2015;82:153-61.

7 Hernandez-Diaz S, Toh S, Cnattingius S. Risk of pre-eclampsia in first and subsequent pregnancies: prospective cohort study. BMJ (Clin Rese ed) 2009;338:b2255.

8 van Rijn BB, Hoeks LB, Bots ML, Franx A, Bruinse HW. Outcomes of subsequent pregnancy after first pregnancy with early-onset preeclampsia. Am J Obstet Gynecol 2006;195:723-8.

9 Chen CW, Jaffe IZ, Karumanchi SA. Pre-eclampsia and cardiovascular disease. Cardiovasc Res 2014;101:579-86.

10 Rodie VA, Freeman DJ, Sattar N, Greer IA. Pre-eclampsia and cardiovascular disease: metabolic syndrome of pregnancy? Atherosclerosis 2004;175:189-202.

11 van Rijn BB, Nijdam ME, Bruinse HW, Roest M, Uiterwaal CS, Grobbee $\mathrm{DE}$, et al. Cardiovascular disease risk factors in women with a history of early-onset preeclampsia. Obstet Gynecol 2013;121:1040-8.

12 Walsh SW. Obesity: a risk factor for preeclampsia. Trends Endocrinol Metab 2007; 18:365-70.

13 Hypertension in Pregnancy. The Management of Hypertensive Disorders During Pregnancy. London: Hypertension in Pregnancy; 2010. 
14 American College of of Gynecologists, Task Force on Hypertension in Pregnancy.. Report of the American College of Obstetricians and Gynecologists' Task Force on hypertension in pregnancy. Obstet Gynecol 2013;122:1122-31.

15 Heida KY, Bots ML, de Groot CJ, van Dunne FM, Hammoud NM, Hoek $A$, et al. Cardiovascular risk management after reproductive and pregnancy-related disorders: a Dutch multidisciplinary evidencebased guideline. Eur J Prev Cardiol 2016;23:1863-79.

16 Piepoli MF, Hoes AW, Agewall S, Albus C, Brotons C, Catapano AL, et al. 2016 European Guidelines on cardiovascular disease prevention in clinical practice: The Sixth Joint Task Force of the European Society of Cardiology and Other Societies on Cardiovascular Disease Prevention in Clinical Practice (constituted by representatives of 10 societies and by invited experts) developed with the special contribution of the European Association for Cardiovascular Prevention \& Rehabilitation (EACPR). Eur Heart J 2016;37:2315-81.

17 Wells GA, Shea B, O'Connell D, Peterson J, Welch V, Losos M, et al. The Newcastle-Ottawa Scale (NOS) for assessing the quality of nonrandomised studies in meta-analyses. 2014 [http://www.ohri.ca/ programs/clinical_epidemiology/oxford.asp] Accessed 11 July 2017

18 Moher D, Liberati A, Tetzlaff J, Altman DG, Group P. Preferred reporting items for systematic reviews and meta-analyses: the PRISMA statement. BMJ (Clinical research ed). 2009;339:b2535.

19 Stroup DF, Berlin JA, Morton SC, Olkin I, Williamson GD, Rennie D, et al. Meta-analysis of observational studies in epidemiology: a proposal for reporting. Meta-analysis Of Observational Studies in Epidemiology (MOOSE) group. JAMA 2000;283:2008-12.

20 Engeland A, Bjorge T, Klungsoyr K, Skjaerven R, Skurtveit S, Furu K. Preeclampsia in pregnancy and later use of antihypertensive drugs. Eur J Epidemiol 2015;30:501-8

21 Lykke JA, Langhoff-Roos J, Sibai BM, Funai EF, Triche EW, Paidas MJ. Hypertensive pregnancy disorders and subsequent cardiovascular morbidity and type 2 diabetes mellitus in the mother. Hypertension (Dallas, Tex : 1979) 2009;53:944-51.

22 Magnussen EB, Vatten LJ, Smith GD, Romundstad PR. Hypertensive disorders in pregnancy and subsequently measured cardiovascular risk factors. Obstet Gynecol 2009;114:961-70.

23 Riise HKR, Sulo G, Tell GS, Igland J, Nygård O, Vollset SE, et al. Incident coronary heart disease after preeclampsia: role of reduced fetal growth, preterm delivery, and parity. I Am Heart Assoc 2017;6:e004158

24 Sibai BM, el-Nazer A, Gonzalez-Ruiz A. Severe preeclampsia-eclampsia in young primigravid women: subsequent pregnancy outcome and remote prognosis. Am J Obstet Gynecol 1986;155:1011-6.

25 Skjaerven R, Wilcox AJ, Klungsoyr K, Irgens LM, Vikse BE, Vatten LJ, et al. Cardiovascular mortality after pre-eclampsia in one child mothers: prospective, population based cohort study. BMJ (Clinical research ed) 2012;345:e7677.

26 Auger N, Fraser WD, Schnitzer M, Leduc L, Healy-Profitos J, Paradis G. Recurrent pre-eclampsia and subsequent cardiovascular risk. Heart (British Cardiac Society) 2017;103:235-43.

27 Gaugler-Senden IP, Berends AL, de Groot CJ, Steegers EA. Severe, very early onset preeclampsia: subsequent pregnancies and future parental cardiovascular health. Eur J Obstet Gynecol Reprod Biol 2008; 140:171-7.

28 Smith GN, Walker MC, Liu A, Wen SW, Swansburg M, Ramshaw H, et al. A history of preeclampsia identifies women who have underlying cardiovascular risk factors. Am J Obstet Gynecol 2009;200:58.e1-8.

29 Ghossein-Doha C, van Neer J, Wissink B, Breetveld NM, de Windt LJ, van Dijk AP, et al. Pre-eclampsia: an important risk factor for asymptomatic heart failure. Ultrasound Obstet Gynecol 2017:49:143-9.
30 Nisell $H$, Lintu $H$, Lunell NO, Mollerstrom G, Pettersson E. Blood pressure and renal function seven years after pregnancy complicated by hypertension. Br J Obstet Gynaecol 1995;102:876-81.

31 Akhter T, Larsson M, Wikstrom AK, Naessen T. Thicknesses of individual layers of artery wall indicate increased cardiovascular risk in severe pre-eclampsia. Ultrasound Obstet Gynecol 2014;43:67580.

32 Ghossein-Doha C, Spaanderman M, Van Kuijk SMJ, Kroon AA, Delhaas $T$, Peeters L. Long-term risk to develop hypertension in women with former preeclampsia: a longitudinal pilot study. Reprod Sci 2014;21:846-53.

33 Kessous R, Shoham-Vardi I, Pariente G, Sergienko R, Sheiner E. Long-term maternal atherosclerotic morbidity in women with preeclampsia. Heart (British Cardiac Society) 2015;101:442-6.

34 Zhang JZ, He J. Risk factors of recurrent preeclampsia and its relation to maternal and offspring outcome. Zhejiang Da Xue Xue Bao Yi Xue Ban 2015:44:258-63.

35 Scholten RR, Lotgering FK, Hopman MT, Van DA, Van dVM, Janssen $M C$, et al. Low plasma volume in normotensive formerly preeclamptic women predisposes to hypertension. Hypertension (Dallas, Tex : 1979) 2015;66:1066-72.

36 Singh MM, Macgillivray I, Mahaffy RG. A study of the long-term effects of pre-eclampsia on blood pressue and renal function. J Obstet Gynaecol 1974;81:903-6.

37 Veerbeek JH, Hermes W, Breimer AY, van RB, Koenen SV, Mol BW, et al. Cardiovascular disease risk factors after early-onset preeclampsia, late-onset preeclampsia, and pregnancy-induced hypertension. Hypertension (Dallas, TX : 1979) 2015;65:600-6.

38 Sibai BM, Mercer B, Sarinoglu C. Severe preeclampsia in the second trimester: recurrence risk and long-term prognosis. Am J Obstet Gynecol 1991;165(5 Pt 1):1408-12.

39 Spaan JJ, Sep SJ, van Balen VL, Spaanderman ME, Peeters LL. Metabolic syndrome as a risk factor for hypertension after preeclampsia. Obstet Gynecol 2012;120(2 Pt 1):311-7.

40 van Oostwaard MF, Langenveld J, Bijloo R, Wong KM, Scholten I, Loix $S$, et al. Prediction of recurrence of hypertensive disorders of pregnancy between 34 and 37 weeks of gestation: a retrospective cohort study. BJOG 2012;119:840-7.

41 Van Oostwaard MF, Langenveld J, Schuit E, Wigny K, Van Susante $\mathrm{H}$, Beune I, et al. Prediction of recurrence of hypertensive disorders of pregnancy in the term period, a retrospective cohort study. Pregnancy Hypertens 2014;4:194-202.

42 Rucker G, Schwarzer G, Carpenter JR, Schumacher M. Undue reliance on $\mathrm{I}(2)$ in assessing heterogeneity may mislead. BMC Med Res Methodol 2008;8:79.

43 Wu P, Haththotuwa R, Kwok CS, Babu A, Kotronias RA, Rushton C, et al. Preeclampsia and future cardiovascular health: a systematic review and meta-analysis. Circ Cardiovasc Qual Outcomes 2017;10: e003497

44 van Rijn BB, Bruinse HW, Veerbeek JH, Post Uiterweer ED, Koenen SV, van der Bom JG, et al. Postpartum circulating markers of inflammation and the systemic acute-phase response after early-onset preeclampsia. Hypertension (Dallas, TX: 1979) 2016;67:404-14.

45 Staff AC, Johnsen GM, Dechend R, Redman CW. Preeclampsia and uteroplacental acute atherosis: immune and inflammatory factors. J Reprod Immunol 2014;101-102:120-6.

46 Veerbeek JH, Brouwers L, Koster MP, Koenen SV, van Vliet EO, Nikkels PG, et al. Spiral artery remodeling and maternal cardiovascular risk: the spiral artery remodeling (SPAR) study. J Hypertens 2016;34:1570-7

47 Benschop L, Duvekot JJ, Versmissen J, van BV, Steegers EAP, Roeters vLJ. Blood pressure profile 1 year after severe preeclampsia. Hypertension (Dallas, TX: 1979) 2018;71:491-8 
Brouwers et al.

48 Groenhof TKJ, van Rijn BB, Franx A, Roeters van Lennep JE, Bots ML, Lely AT. Preventing cardiovascular disease after hypertensive disorders of pregnancy: searching for the how and when. Eur J Prev Cardiol 2017;24:1735-45.

49 Chesley LC, Annitto JE, Cosgrove RA. Prognostic significance of recurrent toxemia of pregnancy. Obstet Gynecol 1964;23:874-81.

50 Funai EF, Friedlander Y, Paltiel O, Tiram E, Xue X, Deutsch L, et al Long-term mortality after preeclampsia. Epidemiology 2005;16:206-15.

51 Ray JG, Booth GL, Alter DA, Vermeulen MJ. Prognosis after maternal placental events and revascularization: PAMPER study. Am J Obstet Gynecol 2016;214:106.e1-.e14.
52 Romundstad PR, Magnussen EB, Smith GD, Vatten LJ. Hypertension in pregnancy and later cardiovascular risk: common antecedents? Circulation 2010:122:579-84

53 Theilen LH, Fraser A, Hollingshaus MS, Schliep KC, Varner MW, Smith $\mathrm{KR}$, et al. All-cause and cause-specific mortality after hypertensive disease of pregnancy. Obstet Gynecol 2016;128:238-44.

54 Wikstrom AK, Haglund B, Olovsson M, Lindeberg SN. The risk of maternal ischaemic heart disease after gestational hypertensive disease. BJOG 2005;112:1486-91. 